\title{
Vaccines and the looming threat of syphilis
}

\author{
Charles T. Ambrose* \\ Department of Microbiology, Immunology, and Molecular Genetics, College of Medicine, University of Kentucky, USA
}

\begin{abstract}
During the past several decades, syphilis has increased in the US and explosively so in China and Russia. While most current cases can be cured by penicillin or second line antibiotics, strains of the syphilis spirochete (Treponema pallidum) resistant to the latter have emerged. And the potential of strains to acquire resistance to penicillin via plasmids has recently been recognized. The 36 million-plus undertreated cases of syphilis in the world represent metaphorically a vast global $37^{\circ} \mathrm{C}$ incubator allowing exchange of antibiotic resistant genes among the human-borne bacteria and theoretically permitting the emergence of penicillin resistant strains. None has yet been identified but they seem inevitable along with the threat of multi-resistant strains.

There is now concern over the lack of new antibiotics to succeed the penicillins. Since such new drugs would likely require a decade or so of research and for federal approval, vaccine-based protection is of immediate consideration here. This approach may exploit the new knowledge about the biology of $T$. pallidum strains their many complex antigens, serotypes, and the latter's distribution in populations. Equally important are recent insights into immunology, such as cellular aspects and antigenic competition.
\end{abstract}

\section{Introduction}

While the prevalence of syphilis in the United States has nearly tripled over the past decade or so, this disease may present an even greater threat to public health in the future [1]. During the past two decades in China there has been 30 -fold increase in syphilis and in Russia nearly a 70 -fold increase. If this 'explosive resurgence' abroad continues and spreads throughout the world, in time it might lead to the emergence of strains of the syphilis spirochete (Treponema pallidum) resistant to penicillin, which has been the standard therapy for the infection since the 1940s. Such deadly strains would eventually reach this country. Resistance to antibiotics (including to penicillin) is present in many bacteria and is transferred among them by genetic means discussed later. Conditions for the appearance of resistant strains of syphilis to penicillin and other 'back-up' antibiotics are especially optimal globally with so many millions of untreated persons harboring multi-drug resistant bacteria. Clinicians are worried about the current absence of new antibiotics to treat many newly resistant human pathogens [2].

This essay reviews briefly the global increase of venereal syphilis and discusses the genetic basis of potential penicillin resistance in $T$. pallidum (Sections $2 \& 3$ ). During the mid-19 $9^{\text {th }}$ century, vaccines were sought for syphilis unsuccessfully, but a century later they seemed unnecessary with the advent of effective antibiotics. However, today with the theoretical threat of antibiotic-resistant syphilis emerging and in the current absence of new successor antibiotics, there is revived interest in vaccines for syphilis.

Treponemal pallidum causes venereal syphilis. Milder, nonvenereal, cutaneous infections are produced by other closely related treponemal species/strains, such as yaws, pinta, and bejel (Sec. 4). The genetic relationship among these four pathogens is treated here. New information about treponemal surface antigens is reviewed next (Sec. 5). Genetic typing of T. pallidum has revealed that multiple strains are prevalent in a human community at any one time. These may compete with one another during a given luetic infection (Sec. 6 \& 8). How to exploit this newer knowledge in devising a vaccine is the challenge at hand.

\section{The recent resurgence of syphilis (Lues venerea)}

In the US before the Second World War, syphilis was estimated to have infected $5 \%$ to $10 \%$ of the US general population and $25 \%$ of impoverished groups -- especially those in southern states. In 1937, US Surgeon General Thomas Parran (1892-1968) had called syphilis the "shadow on the land" [3]. In 1947 the overall prevalence was $66 / 100,000$ but declined dramatically after penicillin became available in the mid-1940s to the level of $2.1 / 100,000$ by 2000 [4]. However, during the so called 'sexual revolution' it rose to $5.5 / 100,000$ by 2013 with $9.3 / 100,000$ in men [1]. A striking example of this increase was the epidemic in San Francisco, where the prevalence was 3.8/100,000 in 1999 and rose to 26/100,000 in 2007 [5] (Table 1).

In China the disease was reported to have been largely eradicated $(<2 / 100,000)$ in the 1960 s by aggressive public health policies of the government. But in recent years there has been an "explosive return of the Great Pox" -- a 25-fold increase from the early 1990s to 2007 and a further 5-fold increase through 2011 [6-8]. The incidence rose to $8.71 / 100,000$ in 2005 throughout the country and to $32 / 100,000$ in 2011. It was much higher in many cities -- e.g., 71/100,000 in Shanghai in 2008 [9]. The one-child policy of the Communist government and female-weighted abortions in families led to an excess of unmarried males and accounted for the rise in MSM (male-sex-male) venereal

Correspondence to: Charles T. Ambrose, Professor, Department of Microbiology, Immunology and Molecular Genetics, College of Medicine, University of Kentucky, USA, Tel: 858-277-3779, Fax: 859-257-8994; E-mail: cambros@uky.edu

Key words: syphilis, vaccine, T. pallidum, antigens, serotypes, antigenic competition Received: December 15, 2015; Accepted: January 12, 2016; Published: January 15,2016 
Table 1. Global Prevalence of Syphilis per 100,000 Population.

\begin{tabular}{|c|c|c|c|c|c|c|c|c|c|c|c|c|}
\hline & 1947 & 1956 & 1988 & 1995 & 1996 & 1999 & 2000 & 2005 & 2007 & 2008 & 2011 & 2013 \\
\hline USA & 66 & 3.9 & & & & 2.1 & & & & & 5.5 & \\
\hline Men & & & & & & & & & & & & 9.3 \\
\hline San Francisco & & & & & & & & 3.8 & & 26 & & \\
\hline China & & $<2$ & & & & & 8.7 & & & 32 & & \\
\hline Shanghai & & & & & & & & & 77 & & & \\
\hline Russia & & & 4 & & 263 & & & & & & & \\
\hline St. Petersburg & & & & 318 & & & & & & & & \\
\hline Finland & & & & 2 & & & & & & & & \\
\hline
\end{tabular}

infections [7]. Migration into crowded cities promoted spread of the disease (Table 1).

In Russia, the collapse of its health system during the 1990s has led a similar rise in syphilis and other infectious diseases -- for syphilis from 4/100,000 in 1988 to 263/100,000 in 1996 [10]. In St. Petersburg the prevalence was 318/100,000 in 1995 [11] (Table 1).

Throughout the world, according to recent estimates, syphilis infects 36 million persons and is newly acquired each year by 11 million more. It causes over 100,000 deaths in spite of penicillin being considered an effective cure for nearly all current luetic infections [1214].

\section{Penicillin and T. pallidum}

Since the mid-1940s, the standard therapy for syphilis has involved various forms of penicillin -- agents containing a critical $\beta$-lactam ring. Syphilitics allergic to penicillin have been treated with secondline antibiotics -- e.g., macrolides (azithromycin, erythromycin, spiramycin) and tetracyclines (doxycycline, etc.) [15]. However, strains of T. pallidum resistant to these second-line antibiotics have appeared -- an example being azithromycin resistance, which was found in $77.3 \%$ of the T. pallidum specimens isolated in 2006 in San Francisco [5].

To date, no penicillin-resistant strains of $T$. pallidum have been reported, but they seem inevitable. The 36 million-plus people infected with $T$. pallidum represent a global microbial pool capable of spreading the syphilis among their billion neighbors. Each person (infected or not) is a $37^{\circ} \mathrm{C}$ incubator harboring in the GI tract and elsewhere a vast array of bacteria which interact with one another -- notably in transferring genetic information via plasmids. These are small pieces of DNA containing genes, including some coding antibiotic resistance. For example, strains of Neisseria gonorrhoeae acquired resistance to penicillin and its derivatives via plasmids encoding $\beta$-lactamase activity which came from ampicillin-resistant strains of Haemophilus influenzae [16]. The enzyme $\beta$-lactamase, inactivates penicillin by disabling its essential $\beta$-lactam ring.

Surprising and disturbing to siphilologists was the discovery in 1981 that plasmids exist in T. pallidum, thus indicating that it has the potential to acquire resistance to penicillin by genetic mechanisms frequently used by other bacteria $[17,18]$. One such example is a strain of Klebsiella pneumoniae (an enteric organism) containing an enzyme which breaks down carbapenem (a $\beta$-lactam antibiotic like penicillin $\mathrm{G})$. The gene for this enzyme is contained in a plasmid, which can move from K. pneumoniae to other bacteria (e.g., T. pallidum). This condition represents "a risk as serious as terrorism" [19].
The ultimate therapeutic threat would be strains of T. pallidum exhibiting resistance to all forms of penicillin and also to backup antibiotics. The medical literature is filled with other examples of multiple drug resistance -- i.e., in staphylococci, enteric bacilli, Mycobacterium tuberculosis, etc. [20]. The World Health Organization and other public health agencies have declared antibiotic resistance to be the greatest threat to global public health [21]. A multiple resistant strain of T. pallidum seems likely to appear somewhere and enter the US population, facilitated by the international travel of infected persons.

A growing concern here is that the "developmental pipeline (for new antibiotic agents) is empty" because many drug companies have found this area unprofitable, focusing instead on drugs treating common chronic diseases. Roche "fled the field in 1999." Eli Lilly "dropped its antibiotic research in 2002," and other companies joined them in abandoning the area [22]. The continued emergence of drugresistant bacteria has prompted the US government "to coax companies back to the field," but the productive momentum they once had may take years to regain.

While new anti-syphilitic drugs would be aggressively sought by the pharmaceutical industry in the event of an epidemic of a resistant strain, there is no great pressure to do so in advance of the therapeutic, commercial demand. Meanwhile, alternate treatments need be considered, such as vaccines. Recent discoveries in the immunology and genetics of the treponemes, extending also to those species/ subspecies of this genus causing non-venereal diseases, may help devise an effective vaccine.

\section{Treponemal diseases in the world}

Siphilologists have been conflicted over relationships among the various treponemal pathogens accounting for four related diseases seen throughout the world: venereal syphilis, yaws, pinta, and bejel (endemic syphilis). All these treponemes are morphologically identical and closely related serologically. All currently are suppressed by penicillin but produce different pathologies, which are summarized below. It's uncertain whether there is any protective cross-reaction among the four agents, but some reciprocal immunity may exist between yaws and syphilis as judged by the latter's relative absence in areas with a high prevalence of yaws [23,24].

Venereal syphilis (VS, lues venerea) elicits genital lesions (chancres) initially and later affects various organ systems as 'tertiary sequelae' in around $30 \%$ of untreated cases. In contrast, three non-venereal treponemal infections produce conspicuous lesions on the exposed parts of the body but no significant constitutional/systemic symptoms (i.e., no chills, fever, weight loss, etc.) and no later organ involvement. 
Yaws (Y) occurs in warm humid rural areas of Africa, SE Asia, and the Americas and causes multiple granular papillomas on unclothed legs, arms, and the face of children mainly. Pinta $(\mathrm{P})$ is present primarily in South/Central America and causes dry scaly lesions on exposed skin. Bejel (B) is found in the dry, warm Middle East, and in severe cases may produce gummatous ulcerative lesion on the face. Bejel in Europe is also termed endemic syphilis. The latter two $(\mathrm{P}, \mathrm{B})$ are characterized also by moist papules in the axilla and skin folds and mucous patches in the mouth.

The characteristic clinical pictures produced by T. pallidum and the non-venereal treponemes reflect the particular tissue tropism of the respective infecting species/strains -- i.e., area of the body primarily affected (tropism, Gr. = 'turning' to some area). The non-venereal treponemes infect mainly the skin and mouth but not the warmer internal organs of the body. For example, unlike venereal syphilis, neither yaws, pinta, nor bejel produces neurological sequelae nor are they transmitted from an infected mother to her foetus [23].

Strain differences may also account for the various clinical pictures seen during late/tertiary stages of venereal venereal syphilis -- i.e., gummatous bony lesions, cardiovascular pathology, or neurological involvement. One particular strain has been found more often in persons with neurosyphilis than in other syphilitics, again suggesting subspecies tropism [24,25].

Medical historians have speculated on how these subtypes evolved and how lues venerea entered into Europe during the mid-1490s. The Columbian theory proposes that this virulent disease arose from a non-venereal treponeme in the Americas and was imported from the Caribbean by infected sailors returning from Columbus' first voyage there. Other theories attribute decaying matter or animals in Africa as the origin of non-venereal treponemes which subsequently infected people and spread via them to the Americas and Europe, ultimately producing lues venerea. Endnote \#1 summarizes such speculations by three investigators which link the various treponemal strains from an evolutionary standpoint [28-30].

K.N. Harper et al. provided scientific support for the Columbian theory of syphilis' entrance into Europe from America based on DNA analyses. This group sequenced select gene regions of twentysix geographically dispersed strains of pathogenic treponemes and concluded that strains causing venereal syphilis now resemble phylogenetically yaws strains from South America [31]. These studies lead logically to a review of treponemal antigens, strain typing, and distribution of the many T. pallidum strains.

\section{Antigens and strain typing of T. pallidum subspp. pallidum}

Subspecies and strain differences reflect in part the complex array of spirochetal surface antigens. Treponemes have a fragile outer membrane and a more stable inner one. The former contains unstable antigenic determinants coded by twelve $\underline{T}$. pallidum repeat genes (Tpr). Different strains express different combinations of Tpr proteins [32]. The inner membrane contains several lipoprotein antigens (e.g., TpN47, TpN17, etc.), which are accessible for expression only when the outer membrane is disrupted or aged. Adding to this complexity is the fact that within one particular antigen (TprK) are seven discrete variable regions coded by different alleles [33]. The relative importance of these different antigens in producing protective immunity is unclear at present, but progress has begun in distinguishing different strains.
Allen Pillay and colleagues developed a method for typing strains of T. pallidum [34]. Intrastrain variability is exhibited by two genes -- a member of the T. pallidum repeat (tpr=Tpr) gene family and the acidic repeat protein (arp) gene. The method involves isolating both genes from a sample, amplifying them by polymerase chain reaction, and analyzing the products of endonuclease digestion. More than 40 strain types have been identified (e.g., 14a, 14d, 15a, etc.) and many subtypes (12df, 14d9, etc.).

Subsequent studies using this new technique have shown that many different strains (up to 16 or so) are present during the same period in a given large urban areas [35]. For example, during 1999-2008, strain type $14 \mathrm{~d} 9$ represented $53 \%$ of the 4 different strains recovered from 69 of persons in San Francisco and 51\% of the 8 found in Seattle $[5,36]$. Strain type 14 a comprised $54 \%$ of the 4 different strains found in Pretoria and $50 \%$ of the 3 in Lisbon [36]. Thus different sexual partners in a community may harbor different strains. To date, so far as I am aware, only one strain has been identified in a given individual, but this may reflect not having examined numerous samples from the same person and perhaps not early enough.

\section{Luetic infections clearance $v s$. reduction $=$ cure $v s$. protection}

Although lacking confirmation, one current supposition is that a given syphilitic may harbor initially several strains of T. pallidum i.e., genetically different subpopulations, each with a wide repertoire of antigens [37]. This diversity could come about in two ways. 1) contacts with different sexual partners would allow the introduction of several different strains from the different 'spirochetal donors'. 2) New antigenic subtypes might also arise de novo by mutations of spirochetes present in an individual.

During the month or so after a person becomes infected from one or more sexual partners and while the immune response is being engaged, many or most of the acquired treponemes are assumed to be cleared, but one or more may persist to produce clinical manifestations $[38,39]$. Of the several concurrent infecting strains of $T$. pallidum, those with stronger or more accessible antigen may produce a 'robust' immune response and be eliminated by opsonic antibodies/phagocytosis and delayed-type immunity, while the 'weaker' strain/s would evoke a lesser response, might escape immune surveillance, and thus survive in the host to produce disease [32]. Whether immunological tolerance might develop to this 'survivor' strain is a consideration. Cullen and Cremer have made a distinction between protection from the disease (nonsterile immunity due merely to reduced spirochetal numbers) and complete clearance of the organism (sterile immunity) [40].

The following is a plausible 'strain' history for an untreated syphilitic, but this scenario has not yet been validated by typing studies. In a given person, an early reduction in strain varieties from immune surveillance may be offset by new strains acquired during later sexual contacts or possibly by mutations in stains already present. Thus the initial repertoire of treponemal strains in that person may change over time.

\section{Studies on syphilis 'vaccination' past and recent}

Philippe Ricord (1800-1889), the famous French venereologist, advocated 'vaccinating' against syphilis in a long series of letters to the Editor of the Union Médicale of Paris, 1850-51. (Endnote\#2) Syphilologists then distinguished between indurated (hardened) and non-indurated chancres and believed that multiple inoculations 
with the "virulent pus" from the latter over many months could be a "prophylaxis against constitutional syphilis" (tertiary sequellae) -much as vaccinia from cowpox pustules protects people from smallpox [41]. A contentious issue at that time was how many sequential contacts with chancre fluid/pus were needed to induce immunity -- some maintained up to a score or more. Proof of immunity for the French investigators then seemed to be merely the later absence of signs of secondary or tertiary syphilis.

Interest in immunizing for syphilis resumed a century later with investigators who sought to obtain non-infectious strains by heating isolates or using other procedures. Their negative results were likely due to labile surface antigens being destroyed in the process. The first limited success was obtained in 1969 by Metzer et al. In earlier studies these investigators had established the protein nature of the immunizing antigens and next sought to attenuate/reduce strain virulence by storage at $4^{\circ} \mathrm{C}$ for 7-10 days. They used a Nichols strain of $T$. pallidum to vaccinate rabbits by IV injections 4-times/week for 7 weeks [42]. Some of the rabbits showed "a high degree of immunity" upon challenge with infectious treponemes -- i.e., no lesions/chancres appeared at the inoculating sites in the skin. But spirochetes were still latent in the treated rabbits -- i.e., "sterile immunity" had not been achieved.

In later, more successful immunization studies, James N. Miller sought to preserve labile surface antigens by using a $\gamma$-irradiated, now non-infectious Nichols strain. He administered this preparation to rabbits IV daily over a 37 -week period and found them "completely resistant to intradermal challenge (with homologous treponemes) administered 10 days after the last immunizing injection" [43]. Protection persisted for one year. There was minimal immunity to challenge with $T$. pertenue, the agent of yaws -- thus indicating specificity for venereal syphilis.

The 37-week long injection protocol in rabbits would not be practical in human subjects. In any case, the prospect for successful immunization is complicated by the possibility that several strains of T. pallidum may infect a given person at the same time. Whether each impedes or influences the immune responses (and the elimination) of others is an issue examined next.

\section{Antigenic competition}

Differences in the immune responses of several concurrently infecting T. pallidum strains are likely due to the 'strength' of their presenting antigens, but another influence might be that of antigenic competition, as explained next. Immunization with two antigens given together (or within days of each other) elicits separate immune responses for both, but the level of each response is lower with paired stimulation than when either antigen is given alone. In other words, two antigen introduced together limit reciprocally somewhat the response of each other. There is considerable variability here, depending on the type of the antigens, the doses, the timing of each stimulus, etc. The nature of this competition was the subject of scores of research papers and reviews in the 1970s [44,45].

My studies in this area then involved lymph node organ cultures prepared from rabbits primed months earlier with two antigens -- e.g., bovine serum albumin and diphtheria toxoid. Each culture consisted of a dozen $1 \mathrm{~mm}^{3}$ sizelymph node fragments bathed in $1 \mathrm{ml}$ of a chemically defined medium [46]. Incubating the cultures with the antigens on Day 0 for two hours elicited the secondary antibody responses to both, which continued over a month as measured in the medium replaced every three days. Pooled lymph nodes from a single primed rabbit provides 60-100 culture tubes, thus allowing many variables (each in 4 or more replicate tubes) to be examined in one experiment -- e,g, detailed dose response effects. The individual responses were measured in the culture medium by hemagglutination titration assays.

The antigenic competition demonstrated in this culture system was not due to some limiting nutritional factor [47]. Related to this phenomenon was the surprising observation that very low levels of certain antibiotics enhanced antibody production in these cultures. For example, adding increasing levels of chloramphenicol or actinomycin $\mathrm{D}$ during the inductive phase of the in vitro response (Days 0-6), produced the expected dose response inhibition during the subsequent productive phase (Days 6-30+). However, extremely low levels of these same agents included after Day 6 cause a paradoxical increase in the antibody responses [46,48].

This observation led to the isolation and characterization of an 'antibody inhibitory material' (AIM) released in the spent medium $[48,49]$. The highest inhibitory effect was found in medium recovered on Day 6, 9, and 12 of the 18-day culture period. I postulated that the paradoxical increase was due to AIM-containing cells producing less AIM because of their being more sensitive to the low levels of actinomycin D than the antibody secreting cells. Other investigators also identified such inhibitory factors (cytokines) and termed them 'suppressor factors' -- i.e., products of so-called 'suppressor' cells (Ts). A decade later, 'T regulatory cells' $\left(\mathrm{T}_{\mathrm{reg}}\right)$ became the preferred term [50].

More recently, $\mathrm{T}_{\text {regs }}$ have been hypothesized to be of two types: one is specific for a given antigen, while another is 'non-specific' but also presumably involved in antigenic competition [50,51]. Examining this phenomenon in terms of antibody production, allergy, and autoimmunity has been the subject of numerous reviews during the past several decades [50-54]. Relevant to the subject of syphilis is whether the multiple antigens of one spirochete strain infecting a person exert antigenic competition with those of other introduced strains.

A 'classical' immunization approach has employed a 'mild' live organism related to a major pathogen in order elicit protective immunity against the latter -- e.g., M. bovis for M. tuberculosis and cowpox or vaccinia virus for the variola virus. Perhaps a relatively 'mild' strain of T. pallidum (like one of the non-luetic treponemes) could be isolated and used as a live vaccine for syphilis, seeking to protect against the more virulent strains. As noted before, there is a "relative absence of venereal syphilis in areas with a high prevalence of yaws" $[23,24]$.

\section{Vaccines and the two forms of immunity}

The focus of a vaccine for syphilis has evolved over the past decade or so. Initially, it has been eliciting a protective humoral response (opsonic antibodies) in order to eliminate T. pallidum via phagocytosis. For example, in 2006 Cullen and Cameron discussed various T.pallidum vaccines -- whole cell, subunits, and non-protein carbohydrate antigens, which might mediate 'opsonophagocytosis' [40].

A seminal paper in 2011 by Carlson et al. viewed the clinical course of syphilis as being determined by the balance between delayed type hypersensitivity (DTH) and humoral immunity to T. pallidum. The authors argued that a strong DTH clears organisms initially from the body, while a strong humoral response later leads to "prolonged infection and progression to tertiary disease" [55]. They concluded that a potential vaccine for syphilis should be directed towards the developing the DTH response. This could be enhanced by including $\mathrm{BCG}$ in the vaccine. 
Finally, in 2014, Cameron and Lukehart emphasized that a successful vaccine should involve both the DTH response and opsonic antibodies/phagocytosis [56]. Thus currently, there seems no consensus for making an effective vaccine.

\section{Summary}

The theoretical threat of a multi-resistant strain of T. pallidum appearing in the near future is compounded by the current absence of new antibiotics effective against any putative new pathogenic strain. Indeed, this therapeutic void prompted a recent article entitled as a question "A return to the pre-antimicrobial era?" [57]. These two linked concerns provided an excuse to review here new information about syphilis (e.g., treponemal antigens, serotyping, etc.) and old relevant studies (e.g., P. Ricord's 'vaccinations', antigenic competition).

Syphilis therapeutics for over a century has been wedded to the concept of a 'magic bullet' (magische Kugel) which kills infecting spirochetes. Modification of some past 'cure' might include, for example, improvement in heavy metal therapy. Fever therapy was effective in relieving the signs and symptoms in some persons with neurosyphilis. A review of pre-antibiotic anti-syphilitic treatments has been submitted elsewhere. But the other important defense against most infections is the host's immune response. Developing a vaccine for syphilis will build on the new information about the biology of $T$. pallidum strains and their immunology. Special consideration must be given to the two competing modalities of immunity -- humoral (opsonization) and cellular (DTH).

\section{Acknowledgements}

I gratefully acknowledge the invaluable assistance of Mrs. Amanda Williams of the Medical Center Library of the University of Kentucky. I am indebted for the continued support of I.S. Tray II.

\section{Endnotes}

\section{Theories on the Origin of Various Treponemal Types}

E.H. Hudson (1961) postulated that the treponemal group arose in Africa from spirochetes growing on decaying vegetable matter [28]. In the beginning, a strain appeared which thrived on the exposed skin of natives, producing the clinical picture of yaws $(\mathrm{Y})$. Around 100,000 years ago their infected descendants migrated to other parts of the world (including the Americas), where a new strain, pinta (P), emerged. In the warm areas of the Middle East, bejel (B) evolved and later spread north. In dry, often cold Europe, where clothing was worn, the spirochete could only survive and spread via the moist genital organs, producing a mild infection called endemic syphilis (ES). Hudson did not explain how virulent venereal syphilis (VS) appeared there around 1494.

C.J. Hackett (1963) proposed a comparable evolution of the treponemes with the difference that that the progenitor of the four was pinta, which crossed over from an animal infection to people about 15,000 BC [29]. It originated in Africa, reached the Americas, and many millennia later spawned yaws there. From pinta in Africa arose bejel, which entered Europe in a mild form (endemic syphilis, ES) beginning in the first century BC. It changed abruptly to the virulent venereal syphilis (VS) near the end of the fifteenth century.

The evolutionary scenarios of Hudson and Hackett both favor the Old World origin for the emergence of venereal syphilis in Europe.

R.S. Morton (1968) advanced a variation in this story: namely, in the later 1490s, morbus gallicus involved "two disease existing sideby side" - the mild endemic syphilis (ES from Africa) long present in Europe and a yaws strain newly introduced from America which evoked an acute illness in the Europeans [30]. For several decades, the severe Columbian (Y) strain dominated as virulent syphilis (VS), but later the milder African strain (ES) became the prevalent one.

2. Philippe Ricord was born in Baltimore of parents who fled there from the French Revolution. During his professional career in Paris, Ricord become the leading physician treating venereal diseases. He is most famous for establishing that syphilis and gonorrhea are distinct diseases -- thus correcting the confusion in this subject created by John Hunter's flawed inoculation experiments with urethral pus.

\section{References}

1. McCarthy M (2014) Syphilis rate rises in the US. BMJ 349: g7756. [Crossref]

2. Denning DW, Bromley MJ (2015) Infectious Disease. How to bolster the antifungal pipeline. Science 347: 1414-1416. [Crossref]

3. Parran T (1937) Shadow on the Land -- Syphilis. New York: Reynal \& Hitchcock: 309

4. Kent ME, Romanelli F (2008) Reexamining syphilis: an update on epidemiology, clinical manifestations, and management. Ann Pharmacother 42: 226-236. [Crossref]

5. Katz KA, Pillay A, Ahrens K, Kohn RP, Hermanstyne K, et al. (2010) Molecular epidemiology of syphilis--San Francisco, 2004-2007. Sex Transm Dis 37: 660-663. [Crossref]

6. Khamsi R (2007) Syphilis rates skyrocket in China. Newscientist Health: 1-2.

7. Hvistendahl M (2012) China. An explosive return of the 'great pox'. Science 335: 390. [Crossref]

8. Gao J, Xu J, Sheng Y, Zhang X, Zhang C, et al. (2013) Increasing trend of syphilis and infection resistance: a retrospective study. Int J Infect Dis 17: e971-976. [Crossref]

9. Zhou P, Li K, Lu H, Qian Y, Gu X, et al. (2010) Azithromycin treatment failure among primary and secondary syphilis patients in Shanghai. Sex Transm Dis 37: 726-729. [Crossref]

10. Tichonova L, Borisenko K, Ward H, Meheus A, Gromyko A, et al. (1997) Epidemics of syphilis in the Russian Federation: trends, origins, and priorities for control. Lancet 350: 210-213. [Crossref]

11. Hilthunen-Back E, Haikala O, Koskela P, Reunala T (1996) Increase of syphilis in Finland related to the Russian epidemic. Euro Surveill 1: 1-2. [Crossref]

12. Cameron CE, Lukehart SA (2014) Current status of syphilis vaccine development: need, challenges, prospects. Vaccine 32: 1602-1609. [Crossref]

13. Lozano R, Naghavi M, Foreman K, Lim S, Shibuya K, et al. (2012) Global and regional mortality from 235 causes of death for 20 age groups in 1990 and 2010: a systematic analysis for the Global Burden of Disease Study 2010. Lancet 380: 20952128. [Crossref]

14. Simms I, Broutet N (2008) Congenital syphilis re-emerging. J Dtsch Dermatol Ges 6 : 269-272. [Crossref]

15. Stamm LV (2010) Global challenge of antibiotic-resistant Treponema pallidum Antimicrob Agents Chemother 54: 583-589. [Crossref]

16. Roberts M, Elwell LP, Falkow S (1977) Molecular characterization of two betalactamase-specifying plasmids isolated from Neisseria gonorrhoeae. J Bacteriol 131: 557-563. [Crossref]

17. Norgard MV, Miller JN (1981) Plasmid DNA in Treponema pallidum (Nichols) potential for antibiotic resistance by syphilis bacteria. Science 213: 553-555. [Crossref]

18. Stapleton JT, Stamm LV, Bassford PJ Jr (1985) Potential for development of antibiotic resistance in pathogenic treponemes. Rev Infect Dis 7 Suppl 2: S314-317. [Crossref]

19. McKenna M (2013) Antibiotic resistance: the last resort. Nature 499: 394-396. [Crossref]

20. Mitruka K, Oeltmann JE, Ijaz K, Haddad MB (2011) Tuberculosis outbreak investigations in the United States, 2002-2008. Emerg Infect Dis 17: 425-431. [Crossref]

21. Reardon S (2014) Antibiotic resistance sweeping developing world. Nature 509: 141 142. [Crossref] 
22. Servick K (2015) The drug push. Science 348: 850-853. [Crossref]

23. Turner TB (1936) The Resistance of Yaws and Syphilis Patients to Reinoculation with Yaws Spirochetes. Am J Hygiene 23: 431-448.

24. Lukehart SA (1985) Prospects for development of a treponemal vaccine. Rev Infect Dis 7 Suppl 2: S305-313. [Crossref]

25. Woods CR (2009) Congenital syphilis-persisting pestilence. Pediatr Infect Dis J 28: 536-537. [Crossref]

26. Tantalo LC, Lukehart SA, Marra CM (2005) Treponema pallidum strain-specific differences in neuroinvasion and clinical phenotype in a rabbit model. J Infect Dis 191: 75-80. [Crossref]

27. Marra C, Sahi S, Tantalo L, Godornes C, Reid T, et al. (2010) Enhanced molecular typing of Treponema pallidum: geographical distribution of strain types and association with neurosyphilis. J Infect Dis 202: 1380-1388. [Crossref]

28. Hudson EH (1961) Historical approach to the terminology of syphilis. Arch Dermatol 84: 545-562. [Crossref]

29. Hackett CJ (1963) On the origin of the human treponematoses (pinta, yaws, endemic syphilis and venereal syphilis). Bull World Health Organ 29: 7-41. [Crossref]

30. Morton RS (1968) Another look at the Morbus Gallicus. Postscript to the meeting of the Medical Society for the Study of Venereal Diseases, Geneva, May 26-28, 1967. Br J Vener Dis 44: 174-177. [Crossref]

31. Harper KN, Ocampo PS, Steiner BM, George RW, Silverman MS, et al. (2008) On the origin of the treponematoses: a phylogenetic approach. PLoS Negl Trop Dis 2: e148. [Crossref]

32. Morgan CA, Lukehart SA, Van Voorhis WC (2003) Protection against syphilis correlates with specificity of antibodies to the variable regions of Treponema pallidum repeat protein K. Infect Immun 71: 5605-5612. [Crossref]

33. Centurion-Lara A, LaFond RE, Hevner K, Godornes C, Molini BJ, et al. (2004) Gene conversion: a mechanism for generation of heterogeneity in the tprK gene of Treponema pallidum during infection. Mol Microbiol 52: 1579-1596. [Crossref]

34. Pillay A, Liu H, Ebrahim S, Chen CY, Lai W, et al. (2002) Molecular typing of Treponema pallidum in South Africa: cross-sectional studies. J Clin Microbiol 40: 256-258. [Crossref]

35. Sutton MY, Liu H, Steiner B, Pillay A, Mickey T, et al. (2001) Molecular subtyping of Treponema pallidum in an Arizona County with increasing syphilis morbidity: use of specimens from ulcers and blood. J Infect Dis 183: 1601-1606. [Crossref]

36. Ho EL, Lukehart SA (2011) Syphilis: using modern approaches to understand an old disease. J Clin Invest 121: 4584-4592. [Crossref]

37. Centurion-Lara A, Godornes C, Castro C, Van Voorhis WC, Lukehart SA (2000) The tprK gene is heterogeneous among Treponema pallidum strains and has multiple alleles. Infect Immun 68: 824-831. [Crossref]

38. LaFond RE, Centurion-Lara A, Godornes C, Rompalo AM, Van Voorhis WC, et al. (2003) Sequence diversity of Treponema pallidum subsp. pallidum tprK in human syphilis lesions and rabbit-propagated isolates. J Bacteriol 185: 6262-6268. [Crossref]

39. Lukehart SA, Shaffer JM, Baker-Zander SA (1992) A subpopulation of Treponema pallidum is resistant to phagocytosis: possible mechanism of persistence. $J$ Infect Dis 166: 1449-1453. [Crossref]
40. Cullen PA, Cameron CE (2006) Progress towards an effective syphilis vaccine: the past, present and future. Expert Rev Vaccines 5: 67-80. [Crossref]

41. Ricord P (1857) Letters on Syphilis: Addressed to the Chief Editor of the Union Médicale ... W.P. Lattimore, trans. Philadelphia: Blanchard and Lea. Letters 32-34. [Crossref]

42. Metzger M, Michalska E, Podwińska J, Smogór W (1969) Immunogenic properties of the protein component of Treponema pallidum. Br J Vener Dis 45: 299-304. [Crossref]

43. Miller JN (1973) Immunity in experimental syphilis. VI. Successful vaccination of rabbits with Treponema pallidum, Nichols strain, attenuated by -irradiation. J Immunol 110: 1206-1215. [Crossref]

44. Taussig MJ (1973) Antigenic competition. Curr Top Microbiol Immunol 60: 125-174 [Crossref]

45. Liacopoulos P, Ben-Efraim S (1975) Antigenic competition. Prog Allergy 18: 97-204 [Crossref]

46. Ambrose CT, Coons AH (1963) Studies on antibody production. VIII. The inhibitory effect of chloramphenicol on the synthesis of antibody in tissue culture. J Exp Med 117 : 1075-1088. [Crossref]

47. Cremer NE (1963) Competition of soluble antigens by tissue culture assay. J Immunol 90: 685-696. [Crossref]

48. Ambrose CT (1969) Regulation of the secondary antibody response in vitro. Enhancement by actinomycin D and inhibition by a macromolecular product of stimulated lymph node cultures. J Exp Med 130: 1003-1029. [Crossref]

49. Ambrose CT (1975) Regulation of the secondary antibody response in vitro. III kkk Inhibitor-containing cells (ICC) of antigen stimulated rabbit lymph node organ cultures. Annales d'Immunologie 126: 3-13.

50. Corthay A (2009) How do regulatory T cells work? Scand J Immunol 70: 326-336. [Crossref]

51. Dwyer JM, Parker D, Turk JL (1981) Suppression of delayed hypersensitivity to tuberculin by antigenic competition. A positive immunoregulatory mechanism sensitive to cyclophosphamide. Immunology 42: 549-559. [Crossref]

52. Basten A, Fazekas de St Groth B (2008) Special regulatory T-cell review: T-cell dependent suppression revisited. Immunology 123: 33-39. [Crossref]

53. Germain RN (2008) Special regulatory T-cell review: A rose by any other name: from suppressor T cells to Tregs, approbation to unbridled enthusiasm. Immunology 123: 20-27. [Crossref]

54. Kapp JA (2008) Special regulatory T-cell review: Suppressors regulated but unsuppressed. Immunology 123: 28-32. [Crossref]

55. Carlson JA, Dabiri G, Cribier B, Sell S (2011) The immunopathobiology of syphilis: the manifestations and course of syphilis are determined by the level of delayed-type hypersensitivity. Am J Dermatopathol 33: 433-460. [Crossref]

56. Cameron CE, Lukehart SA (2014) Current status of syphilis vaccine development need, challenges, prospects. Vaccine 32: 1602-1609. [Crossref]

57. Baker S (2015) Infectious disease. A return to the pre-antimicrobial era? Science 347 1064-1066. [Crossref]

Copyright: (C2016 Ambrose CT. This is an open-access article distributed under the terms of the Creative Commons Attribution License, which permits unrestricted use, distribution, and reproduction in any medium, provided the original author and source are credited. 